\title{
Erratum to: Polyhedral approximation of ellipsoidal uncertainty sets via extended formulations: a computational case study
}

\author{
Andreas Bärmann' ${ }^{1}$ - Andreas Heidt' ${ }^{1}$ - Alexander Martin ${ }^{1}$. \\ Sebastian Pokutta ${ }^{2}$. Christoph Thurner ${ }^{1}$
}

Published online: 24 December 2016

(C) Springer-Verlag Berlin Heidelberg 2016

\section{Erratum to: Comput Manag Sci (2016) 13(2):151-193 DOI 10.1007/s10287-015-0243-0}

The purpose of this erratum is to correct a signing error in the statement of the inner approximation of the second-order cone $\mathbb{L}^{n}$ presented in Bärmann et al. (2016).

In Bärmann et al. (2016), we developed a construction for the inner approximation of $\mathbb{L}^{n}$ based on the ideas of Ben-Tal and Nemirovski (2001) and Glineur (2000). We showed — using the same decomposition as in the aforementioned papers - that it suffices to find an inner approximation of $\mathbb{L}^{2}$, which in turn can be obtained from an inner approximation of the unit ball $\mathbb{B}^{2} \subset \mathbb{R}^{2}$. However, in the statement of the latter two approximations, there was a signing error which we would like to correct here.

The online version of the original article can be found under doi:10.1007/s10287-015-0243-0.

\footnotetext{
$凶$ Andreas Bärmann

andreas.baermann@math.uni-erlangen.de

Andreas Heidt

andreas.heidt@math.uni-erlangen.de

Alexander Martin

alexander.martin@math.uni-erlangen.de

Sebastian Pokutta

sebastian.pokutta@isye.gatech.edu

Christoph Thurner

christoph.thurner@math.uni-erlangen.de

1 Lehrstuhl für Wirtschaftsmathematik, FAU Erlangen-Nürnberg, Erlangen, Germany

2 School of Industrial and Systems Engineering, Georgia Institute of Technology, Atlanta, GA, USA
} 
Our inner approximation of $\mathbb{B}^{2}$ is a regular $m$-gon $\bar{P}_{m}$ inscribed into it. Via an extended formulation, we can state this $m$-gon using a number of variables and constraints logarithmic in $m$ :

Theorem 2.9 The polyhedron

$$
\bar{D}_{k}=\left\{\left(p_{0}, \ldots, p_{k-1}, d_{0}, \ldots, d_{k-1}\right) \in \mathbb{R}^{2 k} \mid \begin{array}{rl}
p_{i-1}=\gamma_{i} p_{i}+\sigma_{i} d_{i}, & (\forall i=1, \ldots, k-1) \\
-d_{i-1} \leq \sigma_{i} p_{i}-\gamma_{i} d_{i}, & (\forall i=1, \ldots, k-1) \\
d_{i-1} \leq \sigma_{i} p_{i}-\gamma_{i} d_{i}, & (\forall i=1, \ldots, k-1) \\
p_{k-1}=\gamma_{k}, & \\
-d_{k-1} \leq \sigma_{k}, & \\
d_{k-1} \leq \sigma_{k} &
\end{array}\right\}
$$

for $k \geq 2$ is an extended formulation for $\bar{P}_{2^{k}}$ with $\operatorname{proj}_{p_{0}, d_{0}}\left(\bar{D}_{k}\right)=\bar{P}_{2^{k}}$.

Proof In the following, we describe the construction of the inner approximation as an iterative procedure. We start by defining the polytope

$$
P_{k-1}:=\left\{\left(p_{k-1}, d_{k-1}\right) \mid p_{k-1}=\gamma_{k},-\sigma_{k} \leq d_{k-1} \leq \sigma_{k}\right\}
$$

Now, we construct a sequence of polytopes $P_{k-1}, P_{k-2}, \ldots, P_{0}$. Assume that polytope $P_{i}$ has already been constructed. In order to obtain polytope $P_{i-1}$ from polytope $P_{i}$, we perform the following actions which we will translate into mathematical operations below:

1. Rotate $P_{i}$ counterclockwise by an angle of $\theta_{i}=\frac{\pi}{2^{i}}$ around the origin to obtain a polytope $P_{i}^{1}$,

2. Reflect $P_{i}^{1}$ at the $x$-axis to obtain a polytope $P_{i}^{2}$,

3. Form the convex hull of $P_{i}^{1}$ and $P_{i}^{2}$ to obtain polytope $P_{i-1}$.

The first step is a simple rotation and can be represented by the linear map

$$
\mathcal{R}_{\theta}: \mathbb{R}^{2} \mapsto \mathbb{R}^{2},\left(\begin{array}{l}
x \\
y
\end{array}\right) \mapsto\left(\begin{array}{cc}
\cos (\theta) & -\sin (\theta) \\
\sin (\theta) & \cos (\theta)
\end{array}\right)\left(\begin{array}{l}
x \\
y
\end{array}\right) .
$$

The reflection at the $x$-axis corresponds to the linear map

$$
\mathcal{M}: \mathbb{R}^{2} \mapsto \mathbb{R}^{2},\left(\begin{array}{l}
x \\
y
\end{array}\right) \mapsto\left(\begin{array}{cc}
1 & 0 \\
0 & -1
\end{array}\right)\left(\begin{array}{l}
x \\
y
\end{array}\right)
$$

Thus, the composition $\mathcal{M R}_{\theta_{i}}$ which first applies $\mathcal{R}_{\theta_{i}}$ and then $\mathcal{M}$, is given by

$$
\mathcal{M R}_{\theta_{i}}: \mathbb{R}^{2} \mapsto \mathbb{R}^{2},\left(\begin{array}{l}
x \\
y
\end{array}\right) \mapsto\left(\begin{array}{cc}
\cos (\theta) & \sin (\theta) \\
\sin (\theta) & -\cos (\theta)
\end{array}\right)\left(\begin{array}{l}
x \\
y
\end{array}\right)
$$

With this, we obtain $P_{i}^{1}=\mathcal{R}_{\theta_{i}}\left(P_{i}\right)$ and $P_{i}^{2}=\left(\mathcal{M} \mathcal{R}_{\theta_{i}}\right)\left(P_{i}\right)$. Finally, adding the two constraints

$$
-d_{i-1} \leq \sigma_{i} p_{i}-\gamma_{i} d_{i}
$$


and

$$
d_{i-1} \leq \sigma_{i} p_{i}-\gamma_{i} d_{i}
$$

yields a polyhedron whose projection onto the variables $\left(d_{i-1}, p_{i-1}\right)$ is $P_{i-1}=$ $\operatorname{conv}\left(P_{i}^{1}, P_{i}^{2}\right)$. Keeping this correspondence in mind, we show that $P_{0}=\bar{P}_{2^{k}}$.

In each iteration, $P_{i}$ is rotated counterclockwise by an angle of $\theta_{i}$ around the origin, such that the vertex of $P_{i}$ with minimal vertical coordinate is rotated to $\left(\gamma_{k}, \sigma_{k}\right)$, therefore $P_{i}^{1}=\mathcal{R}\left(P_{i}\right)$. It is $\left|\mathcal{V}\left(P_{i}^{1}\right)\right|=\left|\mathcal{V}\left(P_{i}\right)\right|$ and $P_{i}^{1}$ lies strictly above the horizontal axis. Applying $\mathcal{M}$, we obtain $P_{i}^{2}=\mathcal{M}\left(P_{i}^{1}\right)$, which satisfies $\left|\mathcal{V}\left(P_{i}^{2}\right)\right|=\left|\mathcal{V}\left(P_{i}^{1}\right)\right|$ and lies strictly below the horizontal axis. Then $P_{i-1}=\operatorname{conv}\left(P_{i}^{1}, P_{i}^{2}\right)$ satisfies $\left|\mathcal{V}\left(P_{i-1}\right)\right|=2\left|\mathcal{V}\left(P_{i}\right)\right|$ because all vertices $v \in \mathcal{V}\left(P_{i}^{1}\right) \cup \mathcal{V}\left(P_{i}^{2}\right)$ remain extreme points of $P_{i}$. We obtain polytope $P_{0}$ after $k-1$ iterations of the above procedure, which has $\left|\mathcal{V}\left(P_{0}\right)\right|=2^{k}$ vertices. As the interior angles at each vertex of $P_{0}$ are of equal size, it follows $P_{0}=\bar{P}_{2^{k}}$. This proves the correctness of our construction.

The intermediate steps of the construction are depicted in Fig. 1 for the case $k=3$, which leads to an octagon-approximation. The upper left picture shows the initial polytope $P_{2}$, which is an interval on the line $x=\gamma_{k}$. The upper middle and upper right picture show its rotation by $45^{\circ}$ counterclockwise and subsequent reflection at the x-axis, thus representing $P_{2}^{1}$ and $P_{2}^{2}$, respectively. The lower left picture shows $P_{1}$ as the convex hull of $P_{2}^{1}$ and $P_{2}^{2}$. The lower middle picture contains both $P_{1}^{1}$ and $P_{1}^{2}$ as a rotation of $P_{1}$ by $90^{\circ}$ counterclockwise and subsequent reflection at the $\mathrm{x}$-axis, respectively. Finally, the lower right picture shows $P_{0}=\bar{P}_{2^{3}}$ as the convex hull of $P_{1}^{1}$ and $P_{1}^{2}$.

By homogenization, we can obtain an inner $\epsilon$-approximation of $\mathbb{L}^{2}$, i.e., a set $\overline{\mathcal{L}}_{\epsilon}^{2}$ with $\left\{(r, x) \in \mathbb{R} \times \mathbb{R}^{2} \mid\|x\| \leq \frac{1}{1+\epsilon} r\right\} \subseteq \overline{\mathcal{L}}^{2} \subseteq \mathbb{L}^{2}$ :

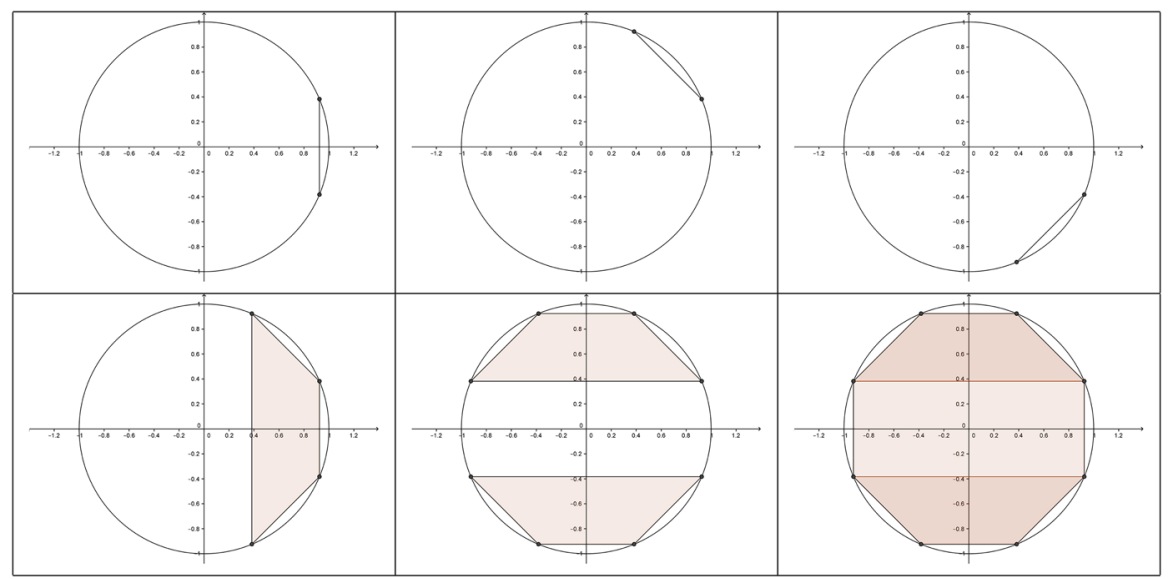

Fig. 1 Construction of the inner approximation of the unit disc $\mathbb{B}^{2}$ for $k=3$ 
Corollary 2.10 The projection of the set

$\overline{\mathcal{L}}_{\epsilon}^{2}=\left\{\left(s, p_{0}, \ldots, p_{k-1}, d_{0}, \ldots, d_{k-1}\right) \in \mathbb{R}^{2 k} \mid \begin{array}{rl}p_{i-1}=\gamma_{i} p_{i}+\sigma_{i} d_{i}, & (\forall i=1, \ldots, k-1) \\ -d_{i-1} \leq \sigma_{i} p_{i}-\gamma_{i} d_{i}, & (\forall i=1, \ldots, k-1) \\ d_{i-1} \leq \sigma_{i} p_{i}-\gamma_{i} d_{i}, & (\forall i=1, \ldots, k-1) \\ p_{k-1}=\gamma_{k} s, & \\ -d_{k-1} \leq \sigma_{k} s, & \\ d_{k-1} \leq \sigma_{k} s & \end{array}\right\}$

with $\epsilon>0$ and $k=\left\lceil\log \left(\pi \arccos \left(\frac{1}{\epsilon+1}\right)^{-1}\right)\right\rceil$ onto the variables $\left(s, p_{0}, d_{0}\right)$ is an inner $\epsilon$-approximation of $\mathbb{L}^{2}$.

We apologize for the incorrect statements of the two approximations in the initial paper.

\section{References}

Ben-Tal A, Nemirovski A (2001) On polyhedral approximations of the second-order cone. Math Oper Res 26:193-205

Bärmann A, Heidt A, Martin A, Pokutta S, Thurner C (2016) Polyhedral approximation of ellipsoidal uncertainty sets via extended formulations: a computational case study. Comput Manag Sci 13(2):151193

Glineur F (2000) Computational experiments with a linear approximation of second-order cone optimization. Image Technical Report 001, Faculté Polytechnique de Mons 